\title{
On the wedge dispiration in an inhomogeneous isotropic nonlinear elastic solid
}

\author{
Arash Yavari* \\ 5 February 2016
}

\begin{abstract}
In this short note we find the stress field of a wedge dispiration (a combination of a screw dislocation and a wedge dislocation along the same line) in an inhomogeneous incompressible isotropic nonlinear solid. We discuss the effect of the radial inhomogeneity of energy function on both the stress field and the energy per unit length of the dispiration and compare with those in an isotropic linear elastic solid.
\end{abstract}

Keywords: Screw Dislocation, Wedge Disclination, Dispiration, Nonlinear elasticity, Defects.

\section{Introduction}

Vito Volterra pioneered the mathematical study of defects in solids in his landmark work [Volterra, 1907] more than a century ago. His work appeared many years before the experimental observation of defects in solids. Volterra classified the line defects into six groups, three translational and three rotational. Love [1927] and Frank [1958] referred to the translational and rotational (line) defects, dislocations and disclinations, respectively. There are only a handful of exact solutions for defects in nonlinear elastic solids in the literature and they are all restricted to homogeneous solids. We should mention Gairola [1979]; Rosakis and Rosakis [1988]; Zubov [1997]; Acharya [2001]; Yavari and Goriely [2012a] for dislocations, Zubov [1997]; Derezin and Zubov [2011]; Yavari and Goriely [2013] for disclinations, and Yavari and Goriely [2012b, 2014] for point defects. Very little is known about the effects of material inhomogeneities on the stress fields and energetics of defects in solids. In the setting of linearized elasticity we are aware of the works of Barnett [1972]; Kroupa [1977]; Lazar [2007]. Both Barnett [1972] and Lazar [2007] assume that shear modulus in

\footnotetext{
*School of Civil and Environmental Engineering \& The George W. Woodruff School of Mechanical Engineering, Georgia Institute of Technology, Atlanta, GA 30332. E-mail: arash.yavari@ce.gatech.edu.
}

a linear elastic solid with a single screw dislocation depends only on a scalar variable. In the cylindrical polar coordinates $(r, \varphi, z)$, Kroupa [1977] considers a hollow circular cylinder with a screw dislocation and assumes that shear modulus depends on $(r, \varphi)$ but the dependence on these two variables is separable.

In the 1950s Kondo [1955a,b] and Bilby, et al. [1955] independently investigated the deep connections between non-Riemannian geometries and the mechanics of defects. In particular, Kondo [1955a] observed that in the presence of defects, the reference configuration, which describes the stress-free state of a solid, is not necessarily Euclidean and referred to the affine connection of this manifold as the material connection. He also realized that the curvature of this connection is a measure of incompatibility, and that the Bianchi identities are conservation equations for incompatibility. Kondo [1955b] showed that torsion tensor is a measure of the density of dislocations. Recently, the geometric theory of solids with distributed defects was revisited and it was demonstrated how to calculate the stress fields of nonlinear solids with distributed defects [Yavari and Goriely, 2012a,b, 2013, 2014].

To the best of our knowledge there are no exact solutions for the stress fields of defects in inhomogeneous nonlinear solids in the literature. A combination of a single screw dislocation and a single wedge disclination along the same line was called a wedge dispiration by Harris [1970]. Ichikawa, et al. [1978] showed that, in the setting of linearized elasticity, a wedge dispiration is a stable defect. Yavari and Goriely [2014] defined a discombination to be any combination of line and point defects in nonlinear solids. In this note we find the stress field of a wedge dispiration in an incompressible isotropic solid with an energy function that explicitly depends on the distance from the dispiration axis. 


\section{Geometric elasticity anelasticity}

In classical nonlinear elasticity one starts with a stressfree reference configuration $\mathcal{B}$ and deformation is a mapping $\varphi: \mathcal{B} \rightarrow \mathcal{S}$, where $\mathcal{S}$ is a Riemannian manifold that here we can assume is the Euclidean space. In the case of defective solids the initial configuration is residually stressed; the classical techniques of nonlinear elasticity cannot be directly used. One approach for analyzing isolated defects is to use Volterra's cutand-weld approach [Rosakis and Rosakis, 1988]. This method, however, cannot be used in the case of distributed defects [Yavari and Goriely, 2012a]. In the geometric formulation of the nonlinear mechanics of defects the stress-free configuration is a Riemannian manifold $(\mathcal{B}, \mathbf{G})$, where $\mathbf{G}$ is the material metric that explicitly depends on the density of defects. One should note that the metric may need to be calculated indirectly, see Yavari and Goriely [2014] for a detailed discussion. In the case of a wedge dispiration the material manifold is calculated directly following Volterra's construction [Tod, 1994; Yavari and Goriely, 2014].

The so-called deformation gradient is the tangent map of $\varphi: \mathcal{B} \rightarrow \mathcal{S}$. It is denoted by $\mathbf{F}=T \varphi$ and at each point $\mathbf{X} \in \mathcal{B}$, it is a linear map $\mathbf{F}(\mathbf{X}): T_{\mathbf{X}} \mathcal{B} \rightarrow T_{\varphi(\mathbf{X})} \mathcal{S}$. Choosing local coordinate charts $\left\{x^{a}\right\}$ and $\left\{X^{A}\right\}$ for $\mathcal{S}$ and $\mathcal{B}$, respectively, $\mathbf{F}$ in components reads

$$
F_{A}^{a}(\mathbf{X})=\frac{\partial \varphi^{a}}{\partial X^{A}}(\mathbf{X})
$$

The adjoint (transpose) of deformation gradient $\mathbf{F}^{\top}$ : $T_{\mathbf{x}} \mathcal{S} \rightarrow T_{\mathbf{X}} \mathcal{B}$ is defined through the relation $\langle\langle\mathbf{F V}, \mathbf{v}\rangle\rangle_{\mathbf{g}}=$ $\left\langle\left\langle\mathbf{V}, \mathbf{F}^{\top} \mathbf{v}\right\rangle_{\mathbf{G}}\right.$, for all $\mathbf{V} \in T_{\mathbf{X}} \mathcal{B}, \mathbf{v} \in T_{\mathbf{X}} \mathcal{S}$ and has components $\left(F^{\boldsymbol{\top}}(\mathbf{X})\right)_{a}^{A}=g_{a b}(\mathbf{x}) F_{B}^{b}(\mathbf{X}) G^{A B}(\mathbf{X})$. The right Cauchy-Green deformation tensor $\mathbf{C}(\mathbf{X})=$ $\mathbf{F}(\mathbf{X})^{\top} \mathbf{F}(\mathbf{X}): T_{\mathbf{X}} \mathcal{B} \rightarrow T_{\mathbf{X}} \mathcal{B}$ has components, $C_{B}^{A}=$ $\left(F^{\mathbf{\top}}\right)^{A}{ }_{a} F^{a}{ }_{B}$. One can show that $\mathbf{C}^{b}=\varphi^{*}(\mathrm{~g})$, i.e. $C_{A B}=\left(g_{a b} \circ \varphi\right) F^{a}{ }_{A} F_{B}^{b}$. The left Cauchy-Green deformation tensor $\mathbf{B}^{\sharp}=\varphi^{*}\left(\mathbf{g}^{\sharp}\right)$ has components $B^{A B}=$ $\left(F^{-1}\right)^{A}{ }_{a}\left(F^{-1}\right)^{B}{ }_{b} g^{a b}$. The spatial analogues of $\mathbf{C}^{b}$ and $\mathbf{B}^{\sharp}$ are

$$
\begin{array}{ll}
\mathbf{c}^{b}=\varphi_{*}(\mathbf{G}), & c_{a b}=\left(F^{-1}\right)^{A}{ }_{a}\left(F^{-1}\right)^{B}{ }_{b} G_{A B}, \\
\mathbf{b}^{\sharp}=\varphi_{*}\left(\mathbf{G}^{\sharp}\right), & b^{a b}=F^{a}{ }_{A} F^{b}{ }_{B} G^{A B} .
\end{array}
$$

$\mathbf{b}^{\sharp}$ is called the Finger deformation tensor. It is straightforward to show that $\mathbf{C}$ and $\mathbf{b}$ have the same principal invariants that are denoted by $I_{1}, I_{2}$, and $I_{3}$ [Marsden and Hughes, 1983]. The Jacobian of deformation gives the Riemannian volume element of the deformed configuration $d v$ in terms of that of the reference configuration $d V$ through the relation $d v=J d V$.
One can show that $J=\sqrt{\operatorname{det} \mathbf{g} / \operatorname{det} \mathbf{G}} \operatorname{det} \mathbf{F}$. For an isotropic solid the strain energy function $W$ only depends on the principal invariants of $\mathbf{b}$, i.e. $W=$ $W\left(I_{1}, I_{2}, I_{3}\right)$ [Ogden, 1984]. For an incompressible solid $I_{3}=J^{2}=1$. It is known that for an incompressible and isotropic solid the Cauchy stress has the classical representation [Truesdell and Noll, 2004; Simo and Marsden, 1983]

$$
\boldsymbol{\sigma}=\left(-p+2 I_{2} \frac{\partial W}{\partial I_{2}}\right) \mathbf{g}^{\sharp}+2 \frac{\partial W}{\partial I_{1}} \mathbf{b}^{\sharp}-2 \frac{\partial W}{\partial I_{2}} \mathbf{b}^{-1},
$$

where $p$ is the Lagrange multiplier corresponding to the incompressibility constraint $J=1$.

\section{Dispiration: Combination of a single screw dislocation and a single wedge disclination along the same line}

In a previous work [Yavari and Goriely, 2014], we constructed the material manifold of a combination of a screw dislocation and a wedge disclination lying on the same line (dispiration). Note that the same problem was analyzed by Zubov [1997] but using a different approach. In these works specific energy functions were used and the body was assumed to be homogeneous. Here, we consider an arbitrary incompressible isotropic solid with an energy function that explicitly depends on the distance from the dispiration axis. This models an axisymmetric inhomogeneous solid with a single wedge dispiration.

Let us denote the Euclidean 3 -space by $\mathcal{B}_{0}$ with the flat metric

$$
d S^{2}=d R_{0}^{2}+R_{0}^{2} d \Theta_{0}^{2}+d Z_{0}^{2},
$$

in the cylindrical coordinates $\left(R_{0}, \Theta_{0}, Z_{0}\right)$. Volterra's cut-and-weld construction of the dispiration is as follows: i) Cut $\mathcal{B}_{0}$ along the closed half planes $\Theta_{0}=0$ and $\Theta_{0}=\omega_{0}\left(0<\omega_{0}<2 \pi\right)$, ii) remove the line $R=0$ and the region $0<\Theta_{0}<\omega_{0}$, iii) translate the two closed half planes by $b_{0}$ in the $Z_{0}$-direction, and iv) identify the two closed half planes. The material metric is written as [Yavari and Goriely, 2014]

$$
\mathbf{G}=\left(\begin{array}{ccc}
1 & 0 & 0 \\
0 & \frac{R^{2}}{\Omega_{0}^{2}}+\frac{b_{0}^{2}}{4 \pi^{2} \Omega_{0}^{2}} & \frac{b_{0}}{2 \pi \Omega_{0}} \\
0 & \frac{b_{0}}{2 \pi \Omega_{0}} & 1
\end{array}\right),
$$

where

$$
\Omega_{0}=\frac{2 \pi}{2 \pi-\omega_{0}} .
$$


Changing the sign of $\omega_{0}$ one obtains a negative disclination, i.e. instead of removing a wedge one inserts in a wedge.

Note that $\operatorname{det} \mathbf{G}=\frac{R^{2}}{\Omega_{0}^{2}}$ and hence the material volume form is: $d V=\sqrt{\operatorname{det} \mathbf{G}} d R d \Theta d Z=\frac{R}{\Omega_{0}} d R d \Theta d Z$. We assume that there are no body forces. We embed the defective body in the Euclidean ambient space $(\mathcal{S}, \mathbf{g})$ and look for solutions of the following form: $(r, \theta, z)=(r(R), \Theta, Z)$. The deformation gradient is written as $\mathbf{F}=\operatorname{diag}\left(r^{\prime}(R), 1,1\right)$ and hence from the incompressibility condition we have

$$
J=\sqrt{\frac{\operatorname{det} \mathbf{g}}{\operatorname{det} \mathbf{G}}} \operatorname{det} \mathbf{F}=\frac{r^{\prime}(R) r(R)}{R / \Omega_{0}}=1 .
$$

Assuming that $r(0)=0$ we have $r=\frac{1}{\sqrt{\Omega_{0}}} R$ and thus
$\mathbf{F}=\operatorname{diag}\left(\frac{1}{\sqrt{\Omega_{0}}}, 1,1\right)$. The Finger tensor has components $b^{a b}=F^{a}{ }_{A} F^{b}{ }_{B} G^{A B}$. For this problem it reads

$$
\mathbf{b}^{\sharp}=\left(\begin{array}{ccc}
\frac{1}{\Omega_{0}} & 0 & 0 \\
0 & \frac{\Omega_{0}^{2}}{R^{2}} & -\frac{b_{0} \Omega_{0}}{2 \pi R^{2}} \\
0 & -\frac{b_{0} \Omega_{0}}{2 \pi R^{2}} & 1+\frac{b_{0}^{2}}{4 \pi^{2} R^{2}}
\end{array}\right) .
$$

The principal invariants of $\mathbf{b}$ ( $\mathbf{b}$ and $\mathbf{C}$ have the same principal invariants) are:

$$
\begin{aligned}
& I_{1}=1+\Omega_{0}+\frac{1}{\Omega_{0}}+\frac{b_{0}^{2}}{4 \pi^{2} R^{2}}, \\
& I_{2}=1+\Omega_{0}+\frac{1}{\Omega_{0}}+\frac{b_{0}^{2}}{4 \pi^{2} R^{2} \Omega_{0}} .
\end{aligned}
$$
Note that $\left(b^{-1}\right)^{a b}$
$g^{a m} g^{b m} c_{m n}$ and hence we have $c^{a b}=$

$$
\mathbf{b}^{-1}=\left(\begin{array}{ccc}
\frac{1}{\Omega_{0}^{2}} & 0 & 0 \\
0 & \frac{\Omega_{0}^{2}}{R^{2}}\left(\Omega_{0}+\frac{b_{0}^{2}}{4 \pi^{2} R^{2}}\right) & -\frac{\Omega_{0} b_{0}}{2 \pi R^{2}}\left(1+\Omega_{0}+\frac{b_{0}^{2}}{4 \pi^{2} R^{2}}\right) \\
0 & -\frac{\Omega_{0} b_{0}}{2 \pi R^{2}}\left(1+\Omega_{0}+\frac{b_{0}^{2}}{4 \pi^{2} R^{2}}\right) & 1+\frac{b_{0}^{2}}{2 \pi^{2} R^{2}}\left(1+\frac{\Omega_{0}}{2}+\frac{b_{0}^{2}}{8 \pi^{2} R^{2}}\right)
\end{array}\right) .
$$

We assume that the body is inhomogeneous, incompressible, and isotropic with an energy function $W=W\left(R, I_{1}, I_{2}\right)$. Note that

$$
\boldsymbol{\sigma}=\left(-p+I_{2} \beta\right) \mathbf{g}^{\sharp}+\alpha \mathbf{b}^{\sharp}-\beta \mathbf{b}^{-1},
$$
has the

$$
\beta(R)=2 \frac{\partial W\left(R, I_{1}, I_{2}\right)}{\partial I_{2}} .
$$

the Cauchy stress following representation

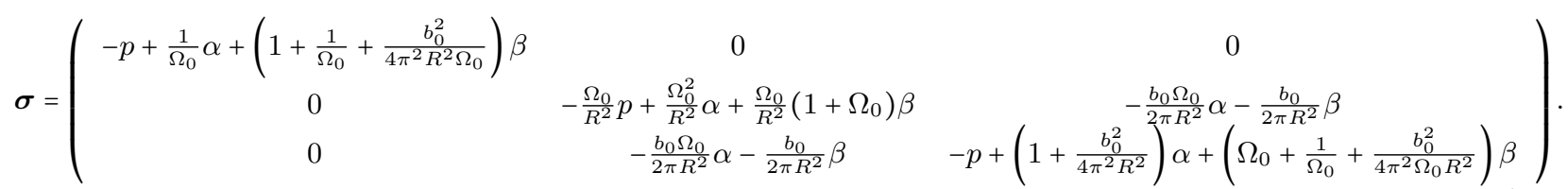

The non-trivial radial equilibrium equation reads $\sigma^{r a}{ }_{\mid a}=\sigma^{r r}{ }_{r}+\frac{1}{r} \sigma^{r r}-r \sigma^{\theta \theta}=0$ (the other two equilibrium equations give us $p=p(R))$. Therefore

$$
\sigma_{, r}^{r r}+\frac{1}{r} \sigma^{r r}-r \sigma^{\theta \theta}=0 .
$$

Or

$$
\begin{aligned}
& \sigma_{, R}^{r r}+\frac{\alpha(R)}{R}\left(\frac{1}{\Omega_{0}}-\Omega_{0}\right) \\
& +\frac{\beta(R)}{R}\left(\frac{1}{\Omega_{0}}-\Omega_{0}+\frac{b_{0}^{2}}{4 \pi R^{2} \Omega_{0}}\right)=0 .
\end{aligned}
$$

This gives a simple ODE for the unknown function $p=$ $p(R)$. Imposing traction-free boundary conditions at 
the boundary $R=R_{o}$, one obtains the Cauchy stress. The non-zero physical components of the Cauchy stress read:

$$
\begin{aligned}
\bar{\sigma}^{r r}(R) & =\left(\frac{1}{\Omega_{0}}-\Omega_{0}\right) \int_{R}^{R_{o}} \frac{\alpha(x)+\beta(x)}{x} d x \\
& +\frac{b_{0}^{2}}{4 \pi \Omega_{0}} \int_{R}^{R_{o}} \frac{\beta(x)}{x^{3}} d x, \\
\bar{\sigma}^{\theta \theta}(R) & =\left(\Omega_{0}-\frac{1}{\Omega_{0}}\right)(\alpha(R)+\beta(R)) \\
& -\frac{b_{0}^{2}}{4 \pi^{2} \Omega_{0}} \frac{\beta(R)}{R^{2}} \\
& +\left(\frac{1}{\Omega_{0}}-\Omega_{0}\right) \int_{R}^{R_{o}} \frac{\alpha(x)+\beta(x)}{x} d x \\
& +\frac{b_{0}^{2}}{4 \pi \Omega_{0}} \int_{R}^{R_{o}} \frac{\beta(x)}{x^{3}} d x, \\
\bar{\sigma}^{z z}(R) & =\left(1-\frac{1}{\Omega_{0}}+\frac{b_{0}^{2}}{4 \pi^{2} R^{2}}\right) \alpha(R) \\
& +\left(\Omega_{0}-1\right) \beta(R) \\
& +\left(\frac{1}{\Omega_{0}}-\Omega_{0}\right) \int_{R}^{R_{o}} \frac{\alpha(x)+\beta(x)}{x} d x \\
& +\frac{b_{0}^{2}}{4 \pi \Omega_{0}} \int_{R}^{R_{o}} \frac{\beta(x)}{x^{3}} d x, \\
\bar{\sigma}^{\theta z}(R) & =-\frac{b_{0} \sqrt{\Omega_{0}}}{2 \pi R}\left(\alpha(R)+\frac{1}{\Omega_{0}} \beta(R)\right) .
\end{aligned}
$$

In the case of a neo-Hookean solid $W=\frac{\mu(R)}{2}\left(I_{1}-3\right)$ and hence $\alpha(R)=\mu(R)$ and $\beta(R)=0$, where $\mu$ is the shear modulus for infinitesimal strains. In this case the dispiration stress field has the following non-zero components:

$$
\begin{aligned}
\bar{\sigma}^{r r}(R) & =\left(\frac{1}{\Omega_{0}}-\Omega_{0}\right) \int_{R}^{R_{o}} \frac{\mu(x)}{x} d x \\
\bar{\sigma}^{\theta \theta}(R) & =\left(\Omega_{0}-\frac{1}{\Omega_{0}}\right) \mu(R) \\
& +\left(\frac{1}{\Omega_{0}}-\Omega_{0}\right) \int_{R}^{R_{o}} \frac{\mu(x)}{x} d x, \\
\bar{\sigma}^{z z}(R) & =\left(1-\frac{1}{\Omega_{0}}+\frac{b_{0}^{2}}{4 \pi^{2} R^{2}}\right) \mu(R) \\
& +\left(\frac{1}{\Omega_{0}}-\Omega_{0}\right) \int_{R}^{R_{o}} \frac{\mu(x)}{x} d x, \\
\bar{\sigma}^{\theta z}(R) & =-\frac{b_{0} \sqrt{\Omega_{0}}}{2 \pi} \frac{\mu(R)}{R} .
\end{aligned}
$$

\subsection{The resultant axial force and en- ergy per unit length of a wedge dispiration}

The resultant force parallel to the dispiration axis is written as

$$
\begin{aligned}
F_{Z} & =\int_{0}^{2 \pi} \int_{0}^{R_{o}} \bar{\sigma}^{z z}(R) \frac{R}{\Omega_{0}} d R d \Theta \\
& =\frac{2 \pi}{\Omega_{0}} \int_{0}^{R_{o}} \bar{\sigma}^{z z}(R) R d R .
\end{aligned}
$$

The energy per unit length of the dispiration reads

$$
\mathcal{W}=\frac{2 \pi}{\Omega_{0}} \int_{0}^{R_{o}} W\left(R, I_{1}, I_{2}\right) R d R
$$

In the case of a wedge disclination both $F_{Z}$ and $\mathcal{W}$ are finite. However, this is not the case, in general, when $b_{0} \neq 0$. For a screw dislocation in the linear approximation $\bar{\sigma}^{z z}(R)=0$ and hence $F_{Z}=0$. There are some well-known subtle issues regarding the energy per unit length in the case of a screw dislocation that we discuss next.

\subsection{Stress field of a wedge disclination}

For a single wedge disclination $\left(b_{0}=0\right)$ we have the following nonzero Cauchy stress components

$$
\begin{aligned}
\bar{\sigma}^{r r}(R) & =\left(\frac{1}{\Omega_{0}}-\Omega_{0}\right) \int_{R}^{R_{o}} \frac{\alpha(x)+\beta(x)}{x} d x, \\
\bar{\sigma}^{\theta \theta}(R) & =\left(\Omega_{0}-\frac{1}{\Omega_{0}}\right)(\alpha(R)+\beta(R)) \\
& +\left(\frac{1}{\Omega_{0}}-\Omega_{0}\right) \int_{R}^{R_{o}} \frac{\alpha(x)+\beta(x)}{x} d x, \\
\bar{\sigma}^{z z}(R) & =\left(1-\frac{1}{\Omega_{0}}\right) \alpha(R)+\left(\Omega_{0}-1\right) \beta(R) \\
& +\left(\frac{1}{\Omega_{0}}-\Omega_{0}\right) \int_{R}^{R_{o}} \frac{\alpha(x)+\beta(x)}{x} d x .
\end{aligned}
$$

Note that in this case $I_{1}=I_{2}=1+\Omega_{0}+\frac{1}{\Omega_{0}}$. 


\subsection{Stress field and energy per unit length of a screw dislocation}

For a single screw dislocation $\left(\Omega_{0}=1\right)$ the non-zero Cauchy stress components read

$$
\begin{aligned}
& \bar{\sigma}^{r r}(R)=\frac{b_{0}^{2}}{4 \pi} \int_{R}^{R_{o}} \frac{\beta(x)}{x^{3}} d x, \\
& \bar{\sigma}^{\theta \theta}(R)=-\frac{b_{0}^{2}}{4 \pi^{2}} \frac{\beta(R)}{R^{2}}+\frac{b_{0}^{2}}{4 \pi} \int_{R}^{R_{o}} \frac{\beta(x)}{x^{3}} d x, \\
& \bar{\sigma}^{z z}(R)=\frac{b_{0}^{2}}{4 \pi^{2}} \frac{\alpha(R)}{R^{2}}+\frac{b_{0}^{2}}{4 \pi} \int_{R}^{R_{o}} \frac{\beta(x)}{x^{3}} d x, \\
& \bar{\sigma}^{\theta z}(R)=-\frac{b_{0}}{2 \pi} \frac{\alpha(R)+\beta(R)}{R} .
\end{aligned}
$$

Note that $I_{1}=I_{2}=3+\frac{b_{0}^{2}}{4 \pi^{2} R^{2}} \geq 3$. It is seen that in the limit of small $b_{0}$ (linear dislocation mechanics) only $\bar{\sigma}^{\theta z}(R)$ is nonzero.

\subsubsection{A few special classes of incompressible isotropic solids}

Next we look at a few special classes of incompressible isotropic solids and the corresponding stress field and energy per unit length of the screw dislocation.

Generalized neo-Hookean solids. For a generalized neo-Hookean solid, $W=W\left(R, I_{1}\right)$ and hence $\beta(R)=0$. Noting that $I_{1} \geq 3$, the Baker-Ericksen inequality reads $\partial W\left(R, I_{1}\right) / \partial I_{1}>0$, and hence $\alpha(R)>0$ [Knowles, 1977]. In this case the non-zero stress components for a screw dislocation read

$$
\begin{aligned}
& \bar{\sigma}^{z z}(R)=\frac{b_{0}^{2}}{4 \pi^{2}} \frac{\alpha(R)}{R^{2}}, \\
& \bar{\sigma}^{\theta z}(R)=-\frac{b_{0}}{2 \pi} \frac{\alpha(R)}{R} .
\end{aligned}
$$

For a screw dislocation in a generalized neo-Hookean solid, the longitudinal force is written as

$$
F_{Z}=\frac{b_{0}^{2}}{2 \pi} \int_{0}^{R_{o}} \frac{\alpha(R)}{R} d R
$$

Neo-Hookean solids. It is known that for a screw dislocation energy per unit length may not be bounded for certain choices of energy functions. For a neoHookean solid

$$
W=\frac{\mu(R)}{2}\left(I_{1}-3\right)=\frac{b_{0}^{2}}{4 \pi^{2}} \frac{\mu(R)}{R^{2}} .
$$

Therefore

$$
\mathcal{W}=\frac{b_{0}^{2}}{2 \pi} \int_{0}^{R_{o}} \frac{\mu(R)}{R} d R
$$

Note that for a neo-Hookean solid from $(3.20) F_{Z}=\mathcal{W}$. For $\mu(R)=\mu_{0}$ it is known that $\mathcal{W}$ cannot be defined due to a logarithmic singularity at $R=0$. The same issue is encountered in linear elasticity [Hirth and Lothe, 1982]. A way out is to remove a core of radius $R_{i}$ (dislocation core) and assume that it has a finite energy $\mathcal{W}_{\text {core. }}$ Energy of the dislocation per unit length in a cylinder of radius $R_{o}$ is then written as (for a homogeneous solid $\left.\mu(R)=\mu_{0}\right)$

$$
\mathcal{W}=\mathcal{W}_{\text {core }}+\frac{b_{0}^{2} \mu_{0}}{2 \pi} \ln \frac{R_{o}}{R_{i}} .
$$

This is the expression of energy per unit length of dislocation for both isotropic linear elastic and neo-Hookean solids. In the case of homogeneous solids Zubov [1997] noticed that for the neo-Hookean energy function $F_{Z}$ is unbounded, which is not physical. However, this is not surprising as the longitudinal force and the energy per unit length are equal for neo-Hookean solids. For an inhomogeneous neo-Hookean solid with $\mu=\mu(R)$, assuming that $\mu(R)=\mu_{0}+O(R)$ as $R \rightarrow 0$, we observe that $\mu(R) / R=\mu_{0} / R+O(1)$ and hence the same singularity is observed in energy per unit length. Note that $\mu(R)>0$, and hence $\mu_{0}>0$.

Power-law materials. Rosakis and Rosakis [1988] considered a screw dislocation in power law materials with the following energy function

$$
W=W\left(I_{1}\right)=\frac{\mu}{2 c}\left\{\left[1+\frac{c}{n}\left(I_{1}-3\right)\right]^{n}-1\right\},
$$

where $\mu, c$, and $n$ are material constants. They showed that for the special case of $n=\frac{1}{2}$ energy per unit length of the screw dislocation is finite. This means that one does not need to remove a core to have a finite energy for this material. One can show that for this material $F_{Z}$ is finite as well.

Hencky material. For an incompressible Hencky solid [Hencky, 1928; Neff, et al., 2014]

$$
\begin{aligned}
& W=\mu\left[\left(\log \lambda_{1}\right)^{2}+\left(\log \lambda_{2}\right)^{2}+\left(\log \lambda_{3}\right)^{2}\right], \\
& \lambda_{1} \lambda_{2} \lambda_{3}=1,
\end{aligned}
$$

where $\lambda_{1}, \lambda_{2}, \lambda_{3}$ are the principal stretches (eigenvalues of $\mathbf{U}=\sqrt{\mathbf{C}}$ ). For the case of a single screw dislocation 
we have

$$
\begin{aligned}
& \lambda_{1}=1+\frac{b_{0}^{2}}{8 \pi^{2} R^{2}}\left(1+\sqrt{1+\frac{16 \pi^{2} R^{2}}{b_{0}^{2}}}\right), \\
& \lambda_{2}=1+\frac{b_{0}^{2}}{8 \pi^{2} R^{2}}\left(1-\sqrt{1+\frac{16 \pi^{2} R^{2}}{b_{0}^{2}}}\right), \\
& \lambda_{3}=1 .
\end{aligned}
$$

For small $R$, the energy density has the following asymptotic expansion

$$
W=2 \mu\left[\ln \left(\frac{b_{0}^{2}}{4 \pi^{2}}\right)-2 \ln R\right]^{2}+O\left(R^{2}\right) \quad \text { as } R \rightarrow 0
$$

and hence energy per unit length of the screw dislocation is finite, i.e., one does not need to remove a core.

For an incompressible exponential Hencky solid [Neff, et al., 2015]

$$
W=\mu e^{\left(\log \lambda_{1}\right)^{2}+\left(\log \lambda_{2}\right)^{2}+\left(\log \lambda_{3}\right)^{2}}, \quad \lambda_{1} \lambda_{2} \lambda_{3}=1 .
$$

One can show that in this case energy per unit length of the dislocation is unbounded.

Remark 3.1. One may conclude that a screw dislocation is a possible defect in incompressible and isotropic solids with the energies (3.24) when $n=\frac{1}{2}$ and (3.25) while it is not a possible defect in the neo-Hookean and exponential Hencky solids. nents

$$
\begin{aligned}
\bar{\sigma}^{r r}(R) & =-\left(\frac{1}{\Omega_{0}}-\Omega_{0}\right) \int_{R_{i}}^{R} \frac{\alpha(x)+\beta(x)}{x} d x \\
& -\frac{b_{0}^{2}}{4 \pi \Omega_{0}} \int_{R_{i}}^{R} \frac{\beta(x)}{x^{3}} d x \\
\bar{\sigma}^{\theta \theta}(R) & =\left(\Omega_{0}-\frac{1}{\Omega_{0}}\right)(\alpha(R)+\beta(R)) \\
& -\frac{b_{0}^{2}}{4 \pi^{2} \Omega_{0}} \frac{\beta(R)}{R^{2}} \\
& -\left(\frac{1}{\Omega_{0}}-\Omega_{0}\right) \int_{R_{i}}^{R} \frac{\alpha(x)+\beta(x)}{x} d x \\
& +\frac{b_{0}^{2}}{4 \pi \Omega_{0}} \int_{R}^{R} \frac{\beta(x)}{x^{3}} d x, \\
\bar{\sigma}^{z z}(R) & =\left(1-\frac{1}{\Omega_{0}}+\frac{b_{0}^{2}}{4 \pi^{2} R^{2}}\right) \alpha(R) \\
& +\left(\Omega_{0}-1\right) \beta(R) \\
& -\left(\frac{1}{\Omega_{0}}-\Omega_{0}\right) \int_{R_{i}}^{R} \frac{\alpha(x)+\beta(x)}{x} d x \\
& -\frac{b_{0}^{2}}{4 \pi \Omega_{0}} \int_{R_{i}}^{R} \frac{\beta(x)}{x^{3}} d x, \\
\bar{\sigma}^{\theta z}(R) & =-\frac{b_{0} \sqrt{\Omega_{0}}}{2 \pi R}\left(\alpha(R)+\frac{1}{\Omega_{0}} \beta(R)\right) .
\end{aligned}
$$

The traction at the outer boundary $R=R_{o}$ reads

$$
\begin{aligned}
\sigma^{r r}\left(R_{o}\right) & =-\left(\frac{1}{\Omega_{0}}-\Omega_{0}\right) \int_{R_{i}}^{R_{o}} \frac{\alpha(x)+\beta(x)}{x} d x \\
& -\frac{b_{0}^{2}}{4 \pi \Omega_{0}} \int_{R_{i}}^{R_{o}} \frac{\beta(x)}{x^{3}} d x .
\end{aligned}
$$

It is seen that the outer boundary cannot be tractionfree, in general. However, for a screw dislocation in a generalized neo-Hookean solid the outer boundary is traction-free as well. Note that energy per unit length of the dispiration is bounded for any material in this case.

\section{Acknowledgments}

\subsection{A hollow solid cylinder with a wedge dispiration}

The author benefited from a discussion with Patrizio Neff. This work was partially supported by NSF Grant No. CMMI 1130856 and AFOSR - Grant No. FA9550-16-1-0003.

\section{References}

us consider a hollow cylinder with inner and outer radii $R_{i}$ and $R_{o}$, respectively. We assume that the interior boundary is traction free, i.e., $\sigma^{r r}\left(R_{i}\right)=0$. In this case instead of (3.13) we obtain a different Cauchy stress field that has the following nonzero physical compo-
Acharya, A. [2001], A model of crystal plasticity based on the theory of continuously distributed dislocations. Journal of the Mechanics and Physics of Solids 49:761-784. 
Barnett, D.M. [1972], On the screw dislocation in an inhomogeneous elastic medium: the case of continuously varying elastic moduli, International Journal of Solids and Structures 8(5): 651-660.

Bilby, B. A., R., Bullough, and E., Smith [1955], Continuous distributions of dislocations: a new application of the methods of non-Riemannian geometry, Proceedings of the Royal Society of London A231(1185): 263-273.

Derezin, S. V. and Zubov, L. M. [2011], Disclinations in Nonlinear Elasticity. ZAMM 91(6):433-442.

Frank, F.C. [1958], On the theory of liquid crystals. Discussions of the Faraday Society 25:19-28.

Gairola, B.K.D. [1979], Nonlinear elastic problems, in Dislocations in Solids, (F.R.N. Nabarro, ed.), vol. 1, Amsterdam, North-Holland.

Harris, W.F. [1970], The dispiration: A distinct new crystal defect of the Weingarten-Volterra type, Philosophical Magazine 22(179): 949-952.

Hencky, H. [1928], Über die Form des Elastizitätsgesetzes bei ideal elastischen Stoffen, Zeitschrift für technische Physik 9: 215220.

Hirth, J.P. and Lothe, J. [1982], Theory of Dislocations, 2nd edition, Krieger, Malabar.

Ichikawa, M., W.F. Harris, and T.-W. Chou [1970], Elastic interaction of dislocations and disclinations, and the elastic energy of dispirations, Materials Science and Engineering 36: 125-132.

Knowles, J.K. [1977], The finite anti-plane shear field near the tip of a crack for a class of incompressible elastic solids, International Journal of Fracture 13(5): 611-639.

Kondo, K. [1955], Geometry of elastic deformation and incompatibility, Memoirs of the Unifying Study of the Basic Problems in Engineering Science by Means of Geometry, (K. Kondo, ed.), vol. 1, Division C, Gakujutsu Bunken Fukyo-Kai, 1955, pp. 5-17.

Kondo, K. [1955], Non-Riemannian geometry of imperfect crystals from a macroscopic viewpoint, Memoirs of the Unifying Study of the Basic Problems in Engineering Science by Means of Geometry, (K. Kondo, ed.), vol. 1, Division D-I, Gakujutsu Bunken FukyoKai, 1955, pp. 6-17

Kroupa, F. [1977], Screw dislocation in a nonhomogeneous medium. Czechoslovak Journal of Physics B 27:1378-1384.

Lazar, M. [2007], On the screw dislocation in a functionally graded material, Mechanics Research Communications 34(3): 305-311.
Love, A.E.H. [1927], A Treatise on the Mathematical Theory of Elasticity, Dover, New York.

Marsden, J.E. and T.J.R. Hughes [1983], Mathematical Foundations of Elasticity, Dover, New York.

Neff, P., Eidel, B., and Martin, R.J. [2014], The axiomatic deduction of the quadratic Hencky strain energy by Heinrich Hencky, arXiv:1402.4027.

Neff, P., Ghiba, I.-D., and Lankeit, J. [2015], The exponentiated Hencky-logarithmic strain energy. Part I: Constitutive issues and rank-one convexity, Journal of Elasticity 121: 143-234.

Ogden, R.W. [1984], Non-Linear Elastic Deformations, Dover, New York.

Rosakis, P. and Rosakis, A. J. [1988], The Screw dislocation problem in incompressible finite elastostatics - a discussion of nonlinear effects. Journal of Elasticity 20(1):3-40.

Simo, J.C. and J.E. Marsden[1983], Stress tensors, Riemannian metrics and the alternative representations of elasticity, Springer LNP 195:369-383.

Tod, K. P. [1994], Conical singularities and torsion. Classical and Quantum Gravity 11:1331-1339.

Truesdell, C. and Noll, W. [2004], The Non-Linear Field Theories of Mechanics, 3rd edition, Springer, Berlin.

Volterra, V. [1907], Sur l'équilibre des corps élastiques multiplement connexes. Annales Scientifiques de l'Ecole Normale Supérieure, Paris 24(3):401-518.

Yavari, A. and A. Goriely [2012] Riemann-Cartan geometry of nonlinear dislocation mechanics. Archive for Rational Mechanics and Analysis 205(1):59-118.

Yavari, A. and A. Goriely [2012] Weyl geometry and the nonlinear mechanics of distributed point defects. Proceedings of the Royal Society A 468:3902-3922.

Yavari, A. and A. Goriely [2013] Riemann-Cartan geometry of nonlinear disclination mechanics. Mathematics and Mechanics of Solids 18(1):91-102.

Yavari, A. and A. Goriely [2014] The geometry of discombinations and its applications to semi-inverse problems in anelasticity. Proceedings of the Royal Society $A$ 470, 2014, 20140403.

Zubov, L.M. [1997], Nonlinear Theory of Dislocations and Disclinations in Elastic Bodies, Springer, Berlin. 